\title{
Existence and Uniqueness of Positive Solutions for a Fractional Switched System
}

\author{
Zhi-Wei Lv ${ }^{1,2}$ and Bao-Feng Chen ${ }^{2}$ \\ ${ }^{1}$ Department of Mathematics, Zhengzhou University, Zhengzhou, Henan 450001, China \\ ${ }^{2}$ Department of Mathematics and Physics, Anyang Institute of Technology, Anyang, Henan 455000, China
}

Correspondence should be addressed to Zhi-Wei Lv; sdlllzw@mail.ustc.edu.cn

Received 25 January 2014; Accepted 11 March 2014; Published 13 April 2014

Academic Editor: Xinan Hao

Copyright (c) 2014 Z.-W. Lv and B.-F. Chen. This is an open access article distributed under the Creative Commons Attribution License, which permits unrestricted use, distribution, and reproduction in any medium, provided the original work is properly cited.

We discuss the existence and uniqueness of positive solutions for the following fractional switched system: $\left({ }^{c} D_{0+}^{\alpha} u(t)+f_{\sigma(t)}(t, u(t))+\right.$ $\left.g_{\sigma(t)}(t, u(t))=0, t \in J=[0,1]\right) ;\left(u(0)=u^{\prime \prime}(0)=0, u(1)=\int_{0}^{1} u(s) d s\right)$, where ${ }^{c} D_{0+}^{\alpha}$ is the Caputo fractional derivative with $2<\alpha \leq 3$, $\sigma(t): J \rightarrow\{1,2, \ldots, N\}$ is a piecewise constant function depending on $t$, and $\mathbb{R}^{+}=[0,+\infty), f_{i}, g_{i} \in C\left[J \times \mathbb{R}^{+}, \mathbb{R}^{+}\right], i=1,2, \ldots, N$. Our results are based on a fixed point theorem of a sum operator and contraction mapping principle. Furthermore, two examples are also given to illustrate the results.

\section{Introduction}

Fractional differential equations arise in various areas of science and engineering. Due to their applications, fractional differential equations have gained considerable attention (cf., e.g., [1-15] and references therein). Moreover, the theory of boundary value problems with integral boundary conditions has various applications in applied fields. For example, heat conduction, chemical engineering, underground water flow, thermoelasticity, and population dynamics can be reduced to the nonlocal problems with integral boundary conditions. In [2], Cabada and Wang considered the following $m$-point boundary value problem for fractional differential equation

$$
\begin{gathered}
{ }^{c} D_{0+}^{\alpha} u(t)+f(t, u(t))=0, \quad 0<t<1, \\
u(0)=u^{\prime \prime}(0)=0, \quad u(1)=\lambda \int_{0}^{1} u(s) d s,
\end{gathered}
$$

where $2<\alpha \leq 3,{ }^{c} D_{0+}^{\alpha}$ is the Caputo fractional derivative, and $f:[0,1] \times[0, \infty) \rightarrow[0, \infty)$ is a continuous function.

On the other hand, a switched system consists of a family of subsystems described by differential or difference equations, which has many applications in traffic control, switching power converters, network control, multiagent consensus, and so forth (see [16-18]). When we consider a switched system, we always suppose that the solution is unique. So it is important to study the uniqueness of solution for a switched system. In [1], Li and Liu investigated the uniqueness of positive solution for the following switched system:

$$
\begin{gathered}
x^{\prime \prime}(t)+f_{\sigma(t)}(t, x(t))=0, \quad t \in J=[0,1], \\
x(0)=0, \quad x(1)=\int_{0}^{1} a(s) x(s) d s,
\end{gathered}
$$

where $\sigma(t): J \rightarrow\{1,2, \ldots, N\}$ is a piecewise constant function depending on $t$, and $\mathbb{R}^{+}=[0,+\infty), f_{i} \in C[J \times$ $\left.\mathbb{R}^{+}, \mathbb{R}^{+}\right], i=1,2, \ldots, N$.

In this paper, we discuss the existence and uniqueness of positive solutions for the following fractional switched system:

$$
\begin{array}{r}
{ }^{c} D_{0+}^{\alpha} u(t)+f_{\sigma(t)}(t, u(t))+g_{\sigma(t)}(t, u(t))=0, \\
t \in J=[0,1], \\
u(0)=u^{\prime \prime}(0)=0, \quad u(1)=\int_{0}^{1} u(s) d s,
\end{array}
$$


where ${ }^{c} D_{0+}^{\alpha}$ is the Caputo fractional derivative with $2<\alpha \leq$ $3, \sigma(t): J \rightarrow\{1,2, \ldots, N\}$ is a piecewise constant function depending on $t$, and $\mathbb{R}^{+}=[0,+\infty), f_{i}, g_{i} \in C\left[J \times \mathbb{R}^{+}, \mathbb{R}^{+}\right]$, $i=1,2, \ldots, N$.

The paper is organized as follows. In Section 2, we present some background materials and preliminaries. Section 3 deals with some existence results. In Section 4, two examples are given to illustrate the results.

\section{Background Materials and Preliminaries}

Definition 1 (see $[3,4]$ ). The fractional integral of order $\alpha$ with the lower limit $t_{0}$ for a function $f$ is defined as

$$
I^{\alpha} f(t)=\frac{1}{\Gamma(\alpha)} \int_{t_{0}}^{t}(t-s)^{\alpha-1} f(s) d s, \quad t>t_{0}, \alpha>0,
$$

where $\Gamma$ is the gamma function.

Definition 2 (see $[3,4])$. For a function $f:[0, \infty) \rightarrow \mathbb{R}$, the Caputo derivative of fractional order is defined as

$$
\begin{aligned}
{ }^{c} D_{0+}^{\alpha} f(t) & =\frac{1}{\Gamma(n-\alpha)} \int_{0}^{t}(t-s)^{n-\alpha-1} f^{(n)}(s) d s \\
\alpha & >0, \quad n=[\alpha]+1 .
\end{aligned}
$$

In the following, let us recall some basic information on cone (see more from $[19,20])$. Let $E$ be a real Banach space and let $P$ be a cone in $E$ which defined a partial ordering in $E$ by $x \leq y$ if and only if $y-x \in P$. $P$ is said to be normal if there exists a positive constant $N$ such that $\theta \leq x \leq y$ implies $\|x\| \leq N\|y\| . P$ is called solid if its interior $\stackrel{\circ}{P}$ is nonempty. If $x \leq y$ and $x \neq y$, we write $x<y$. We say that an operator $A$ is increasing if $x \leq y$ implies $A x \leq A y$.

For all $x, y \in E$, the notation $x \sim y$ means that there exist $\lambda>0$ and $\mu>0$ such that $\lambda x \leq y \leq \mu x$. Clearly, $\sim$ is an equivalence relation. Given $h>\theta$ (i.e., $h \geq \theta$ and $h \neq \theta$ ), we denote by $P_{h}$ the set $P_{h}=\{x \in E \mid x \sim h\}$. It is easy to see that $P_{h} \subset P$.

Definition 3. Let $D=P$ or $D=\stackrel{\circ}{P}$ and let $\gamma$ be a real number with $0 \leq \gamma<1$. An operator $A: P \rightarrow P$ is said to be $\gamma$-concave if it satisfies

$$
A(t x) \geq t^{\gamma} A x, \quad \forall t \in(0,1), x \in D .
$$

Definition 4. An operator $A: E \rightarrow E$ is said to be homogeneous if it satisfies

$$
A(t x)=t A x, \quad \forall t>0, x \in E .
$$

An operator $A: P \rightarrow P$ is said to be subhomogeneous if it satisfies

$$
A(t x) \geq t A x, \quad \forall t \in(0,1), \quad x \in P .
$$

From [2], we have the following result.
Lemma 5. Assume that $2<\alpha \leq 3$ and $f_{i}, g_{i} \in C\left[J \times \mathbb{R}^{+}, \mathbb{R}^{+}\right]$, $i=1,2, \ldots, N$. Then the problem (3) has a solution if and only if $u$ is a solution of the integral equation

$$
u(t)=\int_{0}^{1} G(t, s)\left(f_{\sigma(s)}(s, u(s))+g_{\sigma(s)}(s, u(s))\right) d s,
$$

where

$G(t, s)$

$$
= \begin{cases}\frac{2 t(1-s)^{\alpha-1}(\alpha-1+s)-\alpha(t-s)^{\alpha-1}}{\Gamma(\alpha+1)}, & 0 \leq s \leq t \leq 1, \\ \frac{2 t(1-s)^{\alpha-1}(\alpha-1+s)}{\Gamma(\alpha+1)}, & 0 \leq t \leq s \leq 1 .\end{cases}
$$

Lemma 6. $G(t, s)$ in Lemma 5 has the following property:

(i) $G(t, s)>0$ for all $t, s \in(0,1)$.

(ii) $(1 / \Gamma(\alpha+1)) h(t)(1-s)^{\alpha-1}(\alpha-2+2 s) \leq G(t, s) \leq$ $(2 / \Gamma(\alpha+1)) h(t)(1-s)^{\alpha-1}(\alpha-1+s), t, s \in[0,1], 2<$ $\alpha \leq 3, h(t)=t$.

Proof. From [2], we know that (i) is obvious. For $0 \leq s \leq t \leq$ $1,2<\alpha \leq 3$, we have

$$
\begin{aligned}
2 t(1-s)^{\alpha-1}(\alpha-1+s)-\alpha(t-s)^{\alpha-1} \\
\quad=2 t(1-s)^{\alpha-1}(\alpha-1+s)-\alpha t^{\alpha-1}\left(1-\frac{s}{t}\right)^{\alpha-1} \\
\quad \geq 2 t(1-s)^{\alpha-1}(\alpha-1+s)-\alpha t(1-s)^{\alpha-1} \\
\quad=t(1-s)^{\alpha-1}(\alpha-2+2 s) .
\end{aligned}
$$

This means that (ii) holds.

Theorem 7 (see [19]). Let $P$ be a normal cone in a real Banach space $E, A: P \rightarrow P$ an increasing $\gamma$-concave operator, and $B: P \rightarrow P$ an increasing subhomogeneous operator. Assume that

(i) there is $h>\theta$ such that $A h \in P_{h}$ and $B h \in P_{h}$;

(ii) there exists a constant $\delta_{0}>0$ such that $A x \geq \delta_{0} B x$, $\forall x \in P$.

Then the operator equation $A x+B x=x$ has a unique solution $x^{*}$ in $P_{h}$. Moreover, constructing successively the sequence $y_{n}=$ $A y_{n-1}+B y_{n-1}, n=1,2, \ldots$, for any initial value $y_{0} \in P_{h}$, we have $y_{n} \rightarrow x^{*}$ as $n \rightarrow \infty$.

\section{Main Results}

In this section, we will deal with the existence and uniqueness of positive solutions for problem (3). Let

$$
G_{1}(s, s)=\frac{2}{\Gamma(\alpha+1)}(1-s)^{\alpha-1}(\alpha-1+s) .
$$


It is obvious that

$$
G(t, s) \leq G_{1}(s, s), \quad t, s \in[0,1] .
$$

We consider the Banach space $E=C[[0,1], \mathbb{R}]$ endowed with the norm defined by $\|u\|=\sup _{0 \leq t \leq 1}|u(t)|$. Letting $P=\{u \in$ $E \mid u(t) \geq 0\}$, then $P$ is a cone in $E$. Define an operator $F$ : $E \rightarrow E$ as

$$
(F u)(t)=\int_{0}^{1} G(t, s)\left(f_{\sigma(s)}(s, u(s))+g_{\sigma(s)}(s, u(s))\right) d s .
$$

Then $F$ has a solution if and only if the operator $F$ has a fixed point.

Theorem 8. Let $f_{i}, g_{i} \in C\left[J \times \mathbb{R}^{+}, \mathbb{R}^{+}\right], i=1,2, \ldots, N$. Suppose that the following conditions are satisfied:

$$
\begin{gathered}
\left|f_{i}(t, u(t))-f_{i}(t, v(t))\right| \leq l_{i}(t)|u(t)-v(t)|, \\
\left|g_{i}(t, u(t))-g_{i}(t, v(t))\right| \leq \bar{l}_{i}(t)|u(t)-v(t)|, \\
0<\int_{0}^{1} G_{1}(s, s)\left(l_{i}(s)+\bar{l}_{i}(s)\right) d s<1,
\end{gathered}
$$

where

$$
l_{i}, \bar{l}_{i} \in C\left[J, \mathbb{R}^{+}\right], \quad i=1,2, \ldots, N .
$$

Then the problem (3) has a unique solution on $[0,1]$.

Proof. It follows from Lemma 6 that $F: P \rightarrow P$. For $t \in$ $J, i=1,2, \ldots, N$, we set $\max _{i=1,2, \ldots, N} \sup _{t \in J}\left|f_{i}(t, 0)\right|=M$, $\max _{i=1,2, \ldots, N} \sup _{t \in J}\left|g_{i}(t, 0)\right|=\bar{M}$, and $B_{r}=\left\{u \in C\left[J, \mathbb{R}^{+}\right]:\right.$ $\|u\| \leq r\}$, where

$$
r \geq \frac{(M+\bar{M}) \int_{0}^{1} G_{1}(s, s) d s}{1-\max _{i=1,2, \ldots, N} \int_{0}^{1} G_{1}(s, s)\left(l_{i}(s)+\bar{l}_{i}(s)\right) d s} .
$$

Step 1. We show that $\digamma\left(B_{r}\right) \subset B_{r}$.

For $u \in B_{r}$ and $t \in J, i=1,2, \ldots, N$,

$$
\begin{aligned}
& \int_{0}^{1} G(t, s)\left|f_{i}(s, u(s))+g_{i}(s, u(s))\right| d s \\
& \leq \int_{0}^{1} G_{1}(s, s)\left(\left|f_{i}(s, u(s))-f_{i}(s, 0)\right|+\left|f_{i}(s, 0)\right|\right) d s \\
& \quad+\int_{0}^{1} G_{1}(s, s)\left(\left|g_{i}(s, u(s))-g_{i}(s, 0)\right|+\left|g_{i}(s, 0)\right|\right) d s \\
& \leq r \max _{i=1,2, \ldots, N} \int_{0}^{1} G_{1}(s, s)\left(l_{i}(s)+\bar{l}_{i}(s)\right) d s \\
& \quad+(M+\bar{M}) \int_{0}^{1} G_{1}(s, s) d s \\
& \leq r,
\end{aligned}
$$

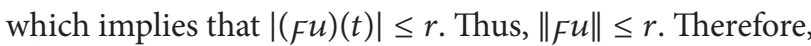

$$
F\left(B_{r}\right) \subset B_{r} .
$$

Step 2. We show that $F$ is a contraction mapping.

For $u, v \in B_{r}$ and for each $t \in J, i=1,2, \ldots, N$, we have

$$
\begin{aligned}
& \int_{0}^{1} G(t, s)\left|f_{i}(s, u(s))-f_{i}(s, v(s))\right| d s \\
& \quad+\int_{0}^{1} G(t, s)\left|g_{i}(s, u(s))-g_{i}(s, v(s))\right| d s \\
& \leq \int_{0}^{1} G_{1}(s, s) l_{i}(s)|u(s)-v(s)| d s \\
& \quad+\int_{0}^{1} G_{1}(s, s) \bar{l}_{i}(s)|u(s)-v(s)| d s \\
& \leq \int_{0}^{1} G_{1}(s, s)\left(l_{i}(s)+\bar{l}_{i}(s)\right) d s\|u-v\| .
\end{aligned}
$$

This, together with $0<\int_{0}^{1} G_{1}(s, s)\left(l_{i}(s)+\bar{l}_{i}(s)\right) d s<1, i=$ $1,2, \ldots, N$, yields that

$$
|(\digamma u)(t)-(\digamma v)(t)| \leq k\|u-v\|,
$$

where

$$
0<k=\max _{i=1,2, \ldots, N} \int_{0}^{1} G_{1}(s, s)\left(l_{i}(s)+\bar{l}_{i}(s)\right) d s<1 .
$$

Thus,

$$
\|\digamma u-\digamma v\| \leq k\|u-v\| .
$$

This means that $F$ is a contraction mapping.

It follows from Banach's contraction mapping that $F$ has a unique fixed point in $B_{r}$. Therefore, the problem (3) has a unique solution.

Corollary 9. Let $f_{i} \in C\left[J \times \mathbb{R}^{+}, \mathbb{R}^{+}\right], i=1,2, \ldots, N$. Suppose that the following conditions are satisfied:

$$
\begin{gathered}
\left|f_{i}(t, u(t))-f_{i}(t, v(t))\right| \leq l_{i}(t)|u(t)-v(t)|, \\
0<\int_{0}^{1} G_{1}(s, s) l_{i}(s) d s<1,
\end{gathered}
$$

where

$$
l_{i} \in C\left[J, \mathbb{R}^{+}\right], \quad i=1,2, \ldots, N .
$$

Then the following fractional switched system

$$
\begin{gathered}
{ }^{c} D_{0+}^{\alpha} u(t)+f_{\sigma(t)}(t, u(t))=0, \quad t \in J=[0,1], \\
u(0)=u^{\prime \prime}(0)=0, \quad u(1)=\int_{0}^{1} u(s) d s,
\end{gathered}
$$

has a unique solution on $[0,1]$.

Theorem 10. Assume that;

$\left(\mathrm{H}_{1}\right) f_{i}, g_{i} \in C\left[J \times \mathbb{R}^{+}, \mathbb{R}^{+}\right]$and $f_{i}(t, x), g_{i}(t, x)$ are increasing in $x$ for $x \in \mathbb{R}^{+}, g_{i}(t, 0) \neq 0, i=1,2, \ldots, N$;

$\left(\mathrm{H}_{2}\right) g_{i}(t, \lambda x) \geq \lambda g_{i}(t, x)$ for $\lambda \in(0,1), \quad t \in J, \quad x \in$ $\mathbb{R}^{+}$, and there exists a constant $\gamma \in(0,1)$ such that $f_{i}(t, \lambda x) \geq \lambda^{\gamma} f_{i}(t, x), \forall t \in J, \lambda \in(0,1), x \in \mathbb{R}^{+}$, $i=1,2, \ldots, N$; 
$\left(\mathrm{H}_{3}\right)$ there exists a constant $\delta_{0}>0$ such that $f_{i}(t, x) \geq$ $\delta_{0} g_{i}(t, x), t \in J, x \in \mathbb{R}^{+}, i=1,2, \ldots, N$.

Then problem (3) has a unique solution $u^{*}$ in $P_{h}$, where $h(t)=$ $t, t \in J$. Moreover, for any initial value $u_{0} \in P_{h}$, constructing successively the sequence

$$
\begin{array}{r}
u_{n+1}(t)=\int_{0}^{1} G(t, s)\left(f_{\sigma(s)}\left(s, u_{n}(s)\right)+g_{\sigma(s)}\left(s, u_{n}(s)\right)\right) d s \\
n=0,1,2, \ldots,
\end{array}
$$

we have $u_{n}(t) \rightarrow u^{*}(t)$ as $n \rightarrow \infty$.

Proof. Define the two operators

$$
\begin{aligned}
& A u(t)=\int_{0}^{1} G(t, s) f_{\sigma(s)}(s, u(s)) d s, \\
& B u(t)=\int_{0}^{1} G(t, s) g_{\sigma(s)}(s, u(s)) d s .
\end{aligned}
$$

From Lemma 6, we have $A: P \rightarrow P$ and $B: P \rightarrow P$. It is obvious that $u$ is the solution of problem (3) if and only if $u=$ $A u+B u$. It follows from $\left(\mathrm{H}_{1}\right)$ that $A$ and $B$ are two increasing operators. Thus, for $u, v \in P, u \geq v$, we have $A u \geq A v$ and $B u \geq B v$.

Step 1. We show that $A$ is a $\gamma$-concave operator and $B$ is a subhomogeneous operator.

In fact, for $\lambda \in(0,1), u \in P, t \in J, i=1,2, \ldots, N$, from $\left(\mathrm{H}_{2}\right)$, we have

$$
\int_{0}^{1} G(t, s) f_{i}(s, \lambda u(s)) d s \geq \lambda^{\gamma} \int_{0}^{1} G(t, s) f_{i}(s, u(s)) d s,
$$

which yields that

$$
A(\lambda u)(t) \geq \lambda^{\gamma} A u(t)
$$

Thus, $A$ is a $\gamma$-concave operator. By a closely similar way, we can see that $B$ is a subhomogeneous operator.

Step 2. We show that $A h \in P_{h}$ and $B h \in P_{h}$.

From Lemma 6 and $\left(\mathrm{H}_{1}\right)$, we have, for $t \in J, i=$ $1,2, \ldots, N$,

$$
\begin{aligned}
& \int_{0}^{1} G(t, s) f_{i}(s, h(s)) d s \\
& \quad \leq \frac{2}{\Gamma(\alpha+1)} h(t) \\
& \quad \times \int_{0}^{1}(1-s)^{\alpha-1}(\alpha-1+s) f_{i}(s, 1) d s, \\
& \int_{0}^{1} G(t, s) f_{i}(s, h(s)) d s
\end{aligned}
$$

$$
\begin{aligned}
& \geq \frac{1}{\Gamma(\alpha+1)} h(t) \\
& \quad \times \int_{0}^{1}(1-s)^{\alpha-1}(\alpha-2+2 s) f_{i}(s, 0) d s .
\end{aligned}
$$

For $i=1,2, \ldots, N$, let

$$
\begin{gathered}
m_{i}=\frac{1}{\Gamma(\alpha+1)} \int_{0}^{1}(1-s)^{\alpha-1}(\alpha-2+2 s) f_{i}(s, 0) d s, \\
\bar{m}_{i}=\frac{2}{\Gamma(\alpha+1)} \int_{0}^{1} 1-s^{\alpha-1}(\alpha-1+s) f_{i}(s, 1) d s .
\end{gathered}
$$

It follows from $g(t, 0) \neq 0$ that $\int_{0}^{1} f_{i}(s, 1) d s \geq \int_{0}^{1} f_{i}(s, 0) d s \geq$ $\delta_{0} \int_{0}^{1} g_{i}(s, 0) d s>0$.

Thus,

$$
m_{i}>0, \quad \bar{m}_{i}>0, \quad i=1,2, \ldots, N .
$$

Letting $\widetilde{m}=\min \left\{m_{i}, i=1,2, \ldots, N\right\}$ and $\widehat{m}=\max \left\{\bar{m}_{i}, i=\right.$ $1,2, \ldots, N\}$, then $\widetilde{m}>0$ and $\widehat{m}>0$. Therefore,

$$
\widetilde{m} h(t) \leq A h(t) \leq \widehat{m} h(t),
$$

which implies that

$$
A h \in P_{h} .
$$

Similarly, we have $B h \in P_{h}$.

Step 3. There exists a constant $\delta_{0}>0$ such that $A u \geq \delta_{0} B u$, $\forall u \in P$.

For $u \in P$ and $t \in J, i=1,2, \ldots, N$, by $\left(\mathrm{H}_{3}\right)$, we have

$$
\int_{0}^{1} G(t, s) f_{i}(s, u(s)) d s \geq \delta_{0} \int_{0}^{1} G(t, s) g_{i}(s, u(s)) d s .
$$

This means that

$$
A u \geq \delta_{0} B u, \quad u \in P .
$$

Therefore, the conditions of Theorem 7 are satisfied. By means of Theorem 7 , we obtain that the operator equation $A u+B u=u$ has a unique solution $u^{*}$ in $P_{h}$. Moreover, for any initial value $u_{0} \in P_{h}$, constructing successively the sequence

$$
\begin{array}{r}
u_{n+1}(t)=\int_{0}^{1} G(t, s)\left(f_{\sigma(s)}\left(s, u_{n}(s)\right)+g_{\sigma(s)}\left(s, u_{n}(s)\right)\right) d s, \\
n=0,1,2, \ldots,
\end{array}
$$

we have $u_{n}(t) \rightarrow u^{*}(t)$ as $n \rightarrow \infty$.

In Theorem 10, if we let $B$ be a null operator, we have the following conclusion.

Corollary 11. Assume that;

$\left(\mathrm{H}_{4}\right) f_{i} \in C\left[J \times \mathbb{R}^{+}, \mathbb{R}^{+}\right]$and $f_{i}(t, x)$ is increasing in $x$ for $x \in \mathbb{R}^{+}, f_{i}(t, 0) \neq 0, i=1,2, \ldots, N$; 
$\left(\mathrm{H}_{5}\right)$ there exists a constant $\gamma \in(0,1)$ such that $f_{i}(t, \lambda x) \geq$ $\lambda^{\gamma} f_{i}(t, x), \forall t \in J, \lambda \in(0,1), x \in \mathbb{R}^{+}, i=1,2, \ldots, N$.

Then the following fractional switched system

$$
\begin{gathered}
{ }^{c} D_{0+}^{\alpha} u(t)+f_{\sigma(t)}(t, u(t))=0, \quad t \in J=[0,1], \\
u(0)=u^{\prime \prime}(0)=0, \quad u(1)=\int_{0}^{1} u(s) d s,
\end{gathered}
$$

has a unique solution $u^{*}$ in $P_{h}$, where $h(t)=t, t \in J$. Moreover, for any initial value $u_{0} \in P_{h}$, constructing successively the sequence

$$
u_{n+1}(t)=\int_{0}^{1} G(t, s) f_{\sigma(s)}\left(s, u_{n}(s)\right) d s, \quad n=0,1,2, \ldots
$$

we have $u_{n}(t) \rightarrow u^{*}(t)$ as $n \rightarrow \infty$.

\section{Examples}

Example 1. Consider the following boundary value problem:

$$
\begin{array}{r}
{ }^{c} D_{0+}^{\alpha} u(t)+f_{\sigma(t)}(t, u(t))+g_{\sigma(t)}(t, u(t))=0, \\
t \in J=[0,1], \\
u(0)=u^{\prime \prime}(0)=0, \quad u(1)=\int_{0}^{1} u(s) d s,
\end{array}
$$

where $\alpha=5 / 2, \sigma(t): J \rightarrow M=\{1,2\}$ is a finite switching signal,

$$
\begin{aligned}
& f_{1}(t, x)=\frac{1}{4(t+2)^{2}} \frac{x}{1+x}+1, \\
& g_{1}(t, x)=\frac{1}{16} \sin ^{2} x+t^{2}, \\
& f_{2}(t, x)=\frac{1}{8(t+2)^{2}} \frac{x}{1+x}+1, \\
& g_{2}(t, x)=\frac{1}{32} \sin ^{2} x+t^{2} .
\end{aligned}
$$

Thus,

$$
f_{i}, g_{i} \in C\left[J \times \mathbb{R}^{+}, \mathbb{R}^{+}\right], \quad i=1,2 .
$$

By computation, we deduce that

$$
\begin{aligned}
& \left|f_{1}\left(t, x_{1}\right)-f_{1}\left(t, x_{2}\right)\right| \leq \frac{1}{16}\left|x_{2}-x_{1}\right|, \\
& \left|g_{1}\left(t, x_{1}\right)-g_{1}\left(t, x_{2}\right)\right| \leq \frac{1}{16}\left|x_{2}-x_{1}\right|,
\end{aligned}
$$

$$
\begin{aligned}
& \left|f_{2}\left(t, x_{1}\right)-f_{2}\left(t, x_{2}\right)\right| \leq \frac{1}{32}\left|x_{2}-x_{1}\right|, \\
& \left|g_{2}\left(t, x_{1}\right)-g_{2}\left(t, x_{2}\right)\right| \leq \frac{1}{32}\left|x_{2}-x_{1}\right| .
\end{aligned}
$$

On the other hand,

$$
\begin{aligned}
\int_{0}^{1} G_{1} & (s, s)\left(l_{1}(s)+\bar{l}_{1}(s)\right) d s \\
& =\int_{0}^{1} \frac{2(1-s)^{(5 / 2)-1}((5 / 2)-1+s)}{\Gamma((5 / 2)+1)}\left(\frac{1}{16}+\frac{1}{16}\right) d s \\
& =\frac{1}{4 \Gamma(7 / 2)} \int_{0}^{1}(1-s)^{3 / 2}\left(\frac{3}{2}+s\right) d s \\
& \leq \frac{1}{4 \Gamma(7 / 2)} \int_{0}^{1}(1-s)^{3 / 2}\left(\frac{3}{2}+1\right) d s \\
& =\frac{1}{3 \sqrt{\pi}} \times \frac{2}{5} \\
& <1
\end{aligned}
$$

$$
\begin{aligned}
\int_{0}^{1} G_{1} & (s, s)\left(l_{1}(s)+\bar{l}_{1}(s)\right) d s \\
& =\int_{0}^{1} \frac{2(1-s)^{(5 / 2)-1}((5 / 2)-1+s)}{\Gamma((5 / 2)+1)}\left(\frac{1}{32}+\frac{1}{32}\right) d s \\
& =\frac{1}{3 \sqrt{\pi}} \times \frac{1}{5} \\
& <1 .
\end{aligned}
$$

Hence, by Theorem 8, BVP (41) has a unique positive solution on $[0,1]$.

Example 2. Consider the following boundary value problem:

$$
\begin{array}{r}
c D_{0+}^{\alpha} u(t)+f_{\sigma(t)}(t, u(t))+g_{\sigma(t)}(t, u(t))=0, \\
t \in J=[0,1], \\
u(0)=u^{\prime \prime}(0)=0, \quad u(1)=\int_{0}^{1} u(s) d s,
\end{array}
$$

where $\alpha=5 / 2, \sigma(t): J \rightarrow\{1,2,3\}$ is a finite switching signal,

$$
\begin{aligned}
& f_{1}(t, x)=x^{1 / 3}+t^{2}+c, \\
& g_{1}(t, x)=\frac{x}{\left(1+t^{2}\right)(1+x)}+b-c, \\
& f_{2}(t, x)=2 x^{1 / 3}+t^{2}+2 c, \\
& g_{2}(t, x)=\frac{2 x}{\left(1+t^{2}\right)(1+x)}+2(b-c),
\end{aligned}
$$




$$
\begin{aligned}
& f_{3}(t, x)=3 x^{1 / 3}+t^{2}+3 c \\
& g_{3}(t, x)=\frac{3 x}{\left(1+t^{2}\right)(1+x)}+3(b-c) .
\end{aligned}
$$

Let $\gamma=1 / 3$ and $0<c<b$. It is obvious that $f_{i}, g_{i} \in$ $C\left[J \times \mathbb{R}^{+}, \mathbb{R}^{+}\right]$and are increasing with respect to the second argument, $g_{i}(t, 0)=b-c>0, i=1,2,3$. On the other hand, for $\lambda \in(0,1), t \in J, x \in[0,+\infty), i=1,2,3$, we have

$$
\begin{aligned}
g_{i}(t, \lambda x) & =\frac{i \lambda x}{\left(1+t^{2}\right)(1+\lambda x)}+i(b-c) \\
& \geq \frac{i \lambda x}{\left(1+t^{2}\right)(1+\lambda x)}+i \lambda(b-c) \\
& =\lambda g_{i}(t, x), \\
f_{i}(t, \lambda x) & =i \lambda^{1 / 3} x^{1 / 3}+t^{2}+i c \\
& \geq \lambda^{1 / 3}\left(i x^{1 / 3}+t^{2}+i c\right) \\
& =\lambda^{\gamma} f_{i}(t, x) .
\end{aligned}
$$

Moreover, for $t \in J, x \in \mathbb{R}^{+}, i=1,2,3$, we have

$$
\begin{aligned}
f_{i}(t, x) & =i x^{1 / 3}+t^{2}+i c \\
& \geq i c \geq \frac{c}{3+(b-c)}(i+i(b-c)) \\
& \geq \frac{c}{3+(b-c)}\left(\frac{i x}{\left(1+t^{2}\right)(1+x)}+i(b-c)\right) \\
& =\delta_{0} g_{i}(t, x),
\end{aligned}
$$

where

$$
\delta_{0}=\frac{c}{3+(b-c)} .
$$

Hence all the conditions of Theorem 10 are satisfied. Thus, $\operatorname{BVP}(46)$ has a unique positive solution in $P_{h}$, where $h(t)=t$, $t \in[0,1]$.

\section{Conflict of Interests}

The authors declare that there is no conflict of interests regarding the publication of this paper.

\section{Acknowledgments}

This research was supported by Henan Province College Youth Backbone Teacher Funds (2011GGJS-213) and the National Natural Science Foundation of China (11271336).

\section{References}

[1] H. T. Li and Y. S. Liu, "On the uniqueness of the positive solution for a second-order integral boundary value problem with switched nonlinearity," Applied Mathematics Letters, vol. 24, no. 12, pp. 2201-2205, 2011.
[2] A. Cabada and G. Wang, "Positive solutions of nonlinear fractional differential equations with integral boundary value conditions," Journal of Mathematical Analysis and Applications, vol. 389, no. 1, pp. 403-411, 2012.

[3] A. A. Kilbas, H. M. Srivastava, and J. J. Trujjllo, Theory and Applications of Fractional Differential Equations, vol. 204 of North-Holland Mathematics Studies, Elsevier, Amsterdam, The Netherlands, 2006.

[4] I. Podlubny, Fractional Differential Equations, Academic Press, New York, NY, USA, 1993.

[5] X. Zhang, L. Liu, and Y. Wu, "The eigenvalue problem for a singular higher order fractional differential equation involving fractional derivatives," Applied Mathematics and Computation, vol. 218, no. 17, pp. 8526-8536, 2012.

[6] Z.-W. Lv, J. Liang, and T.-J. Xiao, "Solutions to the Cauchy problem for differential equations in Banach spaces with fractional order," Computers \& Mathematics with Applications, vol. 62, no. 3, pp. 1303-1311, 2011.

[7] R. P. Agarwal, V. Lakshmikantham, and J. J. Nieto, "On the concept of solution for fractional differential equations with uncertainty," Nonlinear Analysis: Theory, Methods \& Applications, vol. 72, no. 6, pp. 2859-2862, 2010.

[8] R.-N. Wang, T.-J. Xiao, and J. Liang, "A note on the fractional Cauchy problems with nonlocal initial conditions," Applied Mathematics Letters, vol. 24, no. 8, pp. 1435-1442, 2011.

[9] R.-N. Wang, D.-H. Chen, and T.-J. Xiao, "Abstract fractional Cauchy problems with almost sectorial operators," Journal of Differential Equations, vol. 252, no. 1, pp. 202-235, 2012.

[10] J. Henderson and A. Ouahab, "Fractional functional differential inclusions with finite delay," Nonlinear Analysis: Theory, Methods \& Applications, vol. 70, no. 5, pp. 2091-2105, 2009.

[11] V. Lakshmikantham, "Theory of fractional functional differential equations," Nonlinear Analysis: Theory, Methods \& Applications, vol. 69, no. 10, pp. 3337-3343, 2008.

[12] V. Lakshmikantham and A. S. Vatsala, "Basic theory of fractional differential equations," Nonlinear Analysis: Theory, Methods \& Applications, vol. 69, no. 8, pp. 2677-2682, 2008.

[13] F. Li, "Mild solutions for fractional differential equations with nonlocal conditions," Advances in Difference Equations, Article ID 287861, 9 pages, 2010.

[14] X. Q. Zhang, "Positive solution for a class of singular semipositone fractional differential equations with integral boundary conditions," Boundary Value Problems, vol. 2012, article 123, 2012.

[15] C. Yang and C. Zhai, "Uniqueness of positive solutions for a fractional differential equation via a fixed point theorem of a sum operator," Electronic Journal of Differential Equations, vol. 2012, no. 70, pp. 1-8, 2012.

[16] A. A. Agrachev and D. Liberzon, "Lie-algebraic stability criteria for switched systems," SIAM Journal on Control and Optimization, vol. 40, no. 1, pp. 253-269, 2001.

[17] J. Daafouz, P. Riedinger, and C. Iung, "Stability analysis and control synthesis for switched systems: a switched Lyapunov function approach," IEEE Transactions on Automatic Control, vol. 47, no. 11, pp. 1883-1887, 2002.

[18] L. Gurvits, R. Shorten, and O. Mason, "On the stability of switched positive linear systems," IEEE Transactions on Automatic Control, vol. 52, no. 6, pp. 1099-1103, 2007. 
[19] C. Zhai and D. R. Anderson, "A sum operator equation and applications to nonlinear elastic beam equations and LaneEmden-Fowler equations," Journal of Mathematical Analysis and Applications, vol. 375, no. 2, pp. 388-400, 2011.

[20] D. J. Guo and V. Lakshmikantham, Nonlinear Problems in Abstract Cone, vol. 5, Academic Press, San Diego, Calif, USA, 1988. 


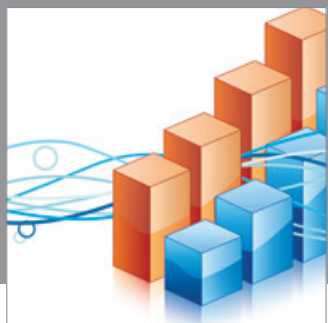

Advances in

Operations Research

mansans

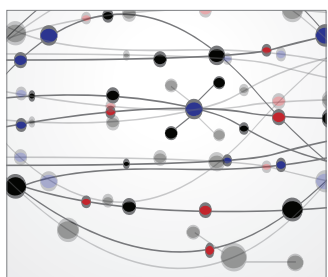

The Scientific World Journal
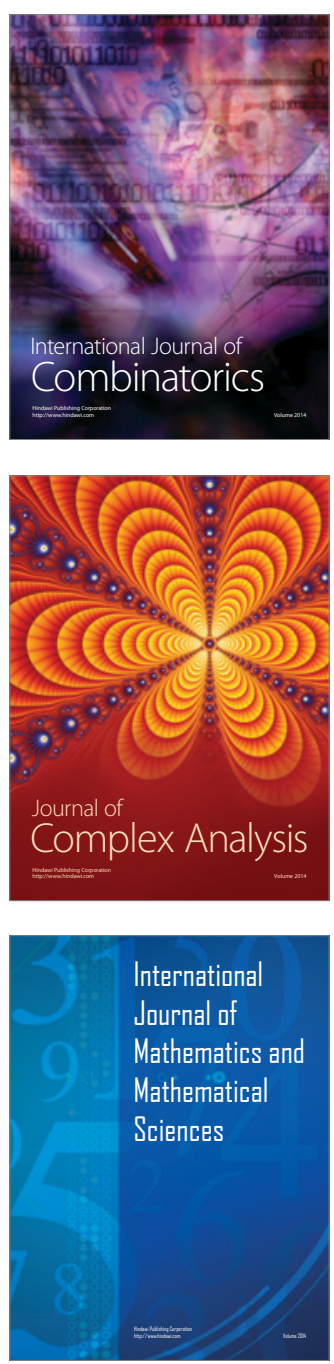
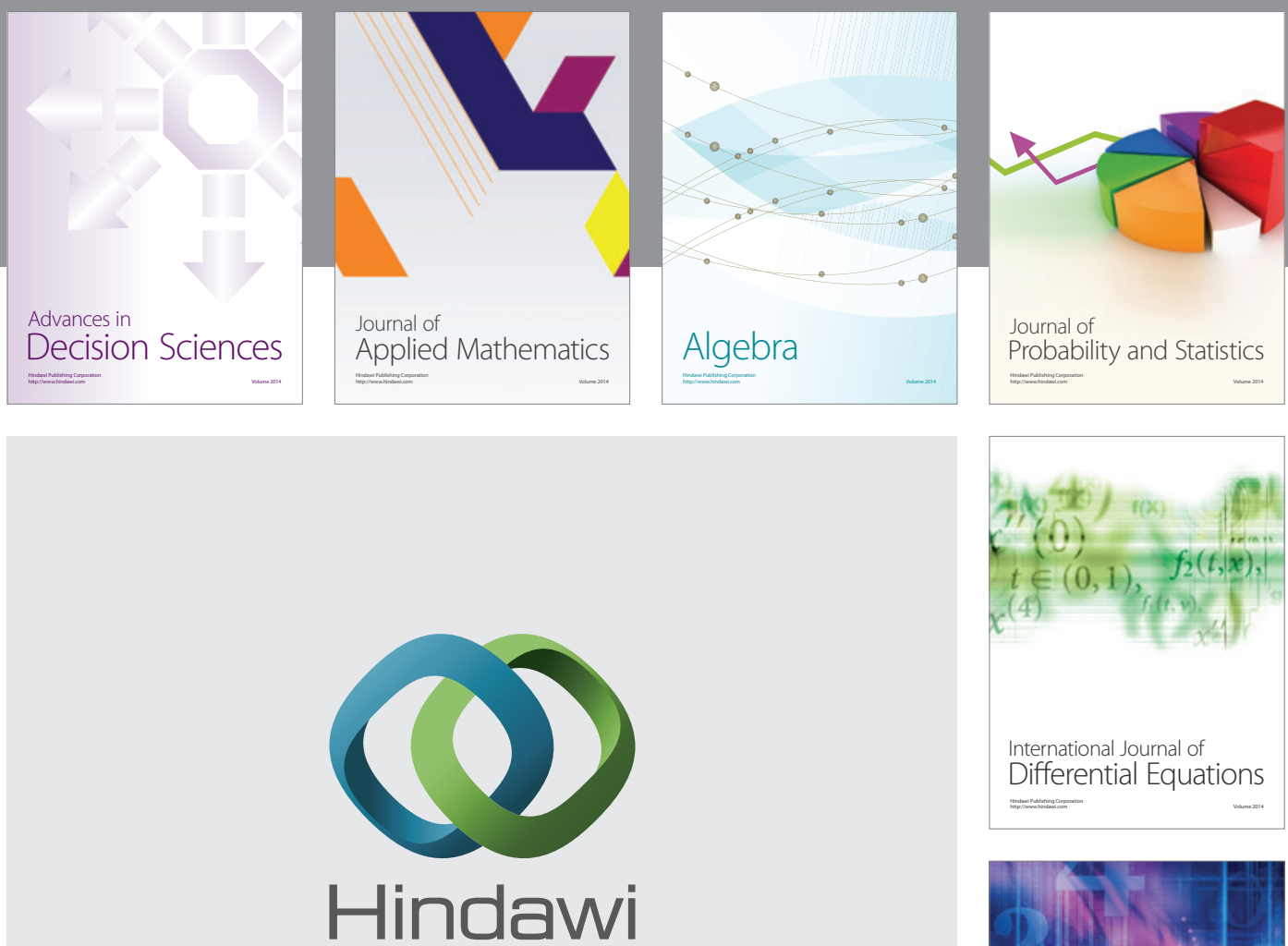

Submit your manuscripts at http://www.hindawi.com
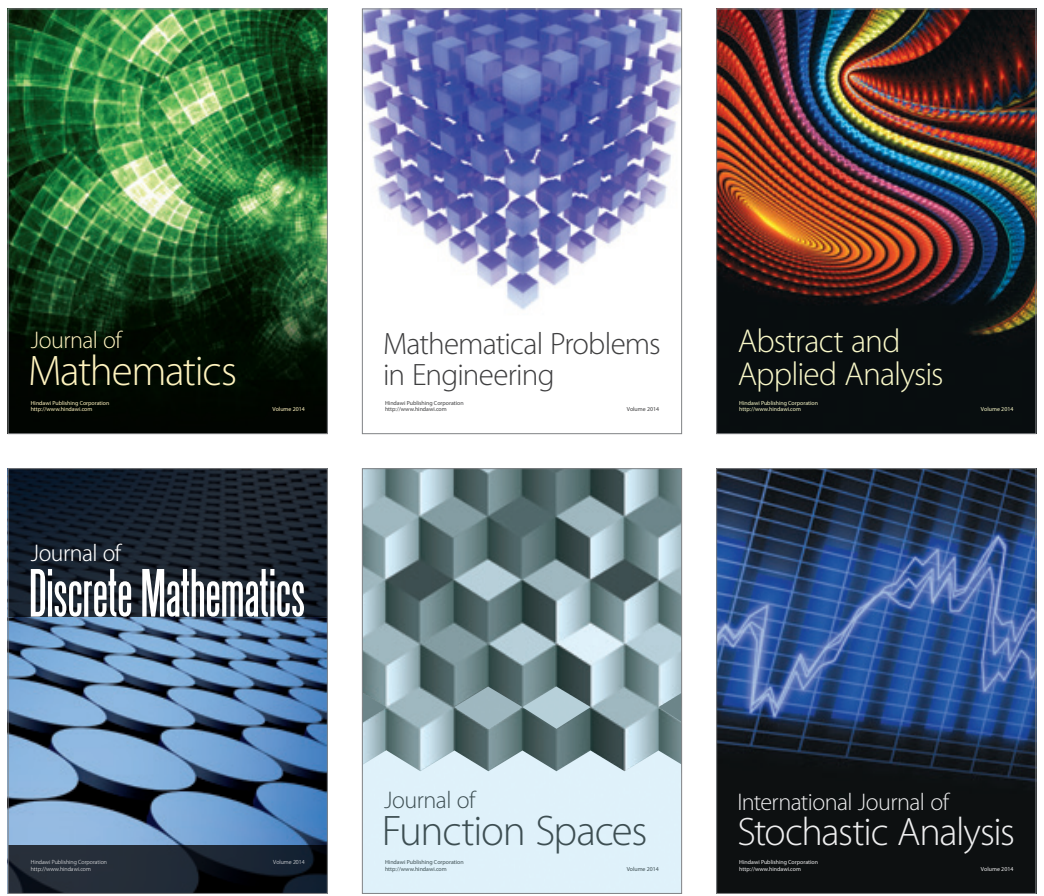

Journal of

Function Spaces

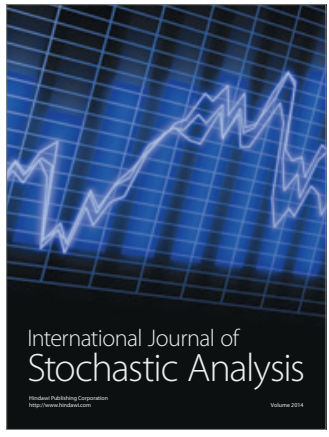

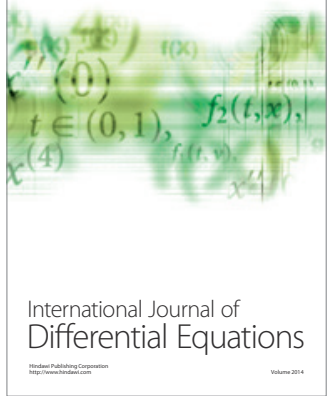
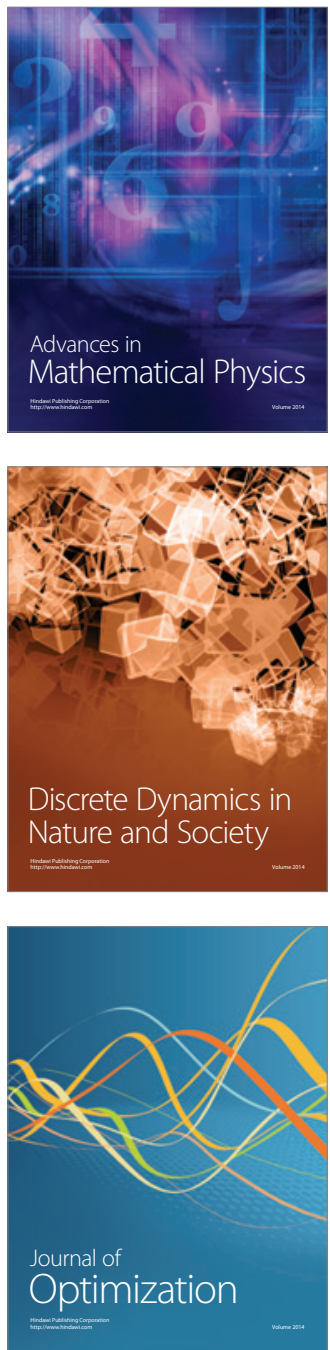